\title{
$\nabla$
}

\section{Vemurafenib and cutaneous adverse events - report of five cases*}

\author{
Giselle de Barros Silva ${ }^{1}$ \\ Mariana Petaccia de Macedo ${ }^{1}$ \\ Ivana Lameiras Gibbons ${ }^{1}$
}

\author{
Adriana Pessoa Mendes ${ }^{1}$ \\ Clóvis Antônio Lopes Pinto \\ João Pedreira Duprat Neto ${ }^{1}$
}

DOI: http:/ / dx.doi.org/10.1590/abd1806-4841.20153841

\begin{abstract}
A bstract: Vemurafenib is a selective inhibitor of V600E-mutant BRAF protein used to treat metastatic and unresectable melanoma. Clinical trials have shown increased overall survival and progression-free survival in patients treated with Vemurafenib. However, cutaneous adverse events are common during treatment. We report five cases of metastatic melanoma with BRAF V600E positivity, treated with Vemurafenib and its cutaneous adverse events. Dermatologists and oncologists need to be aware of possible skin changes caused by this medication, which is increasingly employed in melanoma treatment. Monitoring of patients during therapy is important for early treatment of adverse cutaneous cutaneous adverse events, improvement in quality of life and adherence to treatment.
\end{abstract}

Keywords: Drug eruptions; Drug therapy; Melanoma

\section{INTRODUCTION}

Vemurafenib selectively inhibits the BRAF V600E mutant protein. It is used in the treatment of unresectable or metastatic melanoma with increased overall survival and progression-free survival. ${ }^{1}$

BRAF mutation is the most common in human cancers (found in $40-60 \%$ of skin melanomas). ${ }^{1,2}$ Vemurafenib inhibits BRAF V600E, blocking the MAPK pathway and preventing tumor cell proliferation. ${ }^{1,2}$

Cutaneous adverse events are common during treatment and include: photosensitivity, keratosis pilaris-like rash, plantar hyperkeratosis, warts, cutaneous squamous cell carcinoma (SCC) and keratoacanthoma. ${ }^{1,3}$ There have been reports of darkening and eruption of new nevi and second primary melanoma, panniculitis and skin sensitivity or dermatitis by radiation recall on irradiated skin. ${ }^{4-9}$

In this article, we report five cases of BRAF V600E metastatic melanoma treated with Vemurafenib, revealing cutaneous adverse events associated with treatment.

\section{CASE REPORT \\ Case 1}

Female patient, aged 66, was diagnosed with nodular melanoma on her left leg in 2008. In 2010, intransit metastases were resected. She was treated with IFN and ipilimumab, with no response. Vemurafenib was administered in February 2013. She endured photosensitivity, keratoacanthoma, actinic keratosis and alopecia (Figure 1). After 6 months, treatment was interrupted due to disease progression.

\section{Case 2}

Male patient, aged 76, was diagnosed with superficial spreading melanoma on the left arm in July 2009. In 2012, he developed metastases. In March 2013, he was started on Vemurafenib. After 4 months, he developed well-differentiated SCC on the left forearm (Figure 2). In September 2013, Vemurafenib was suspended due to acute respiratory symptoms. 


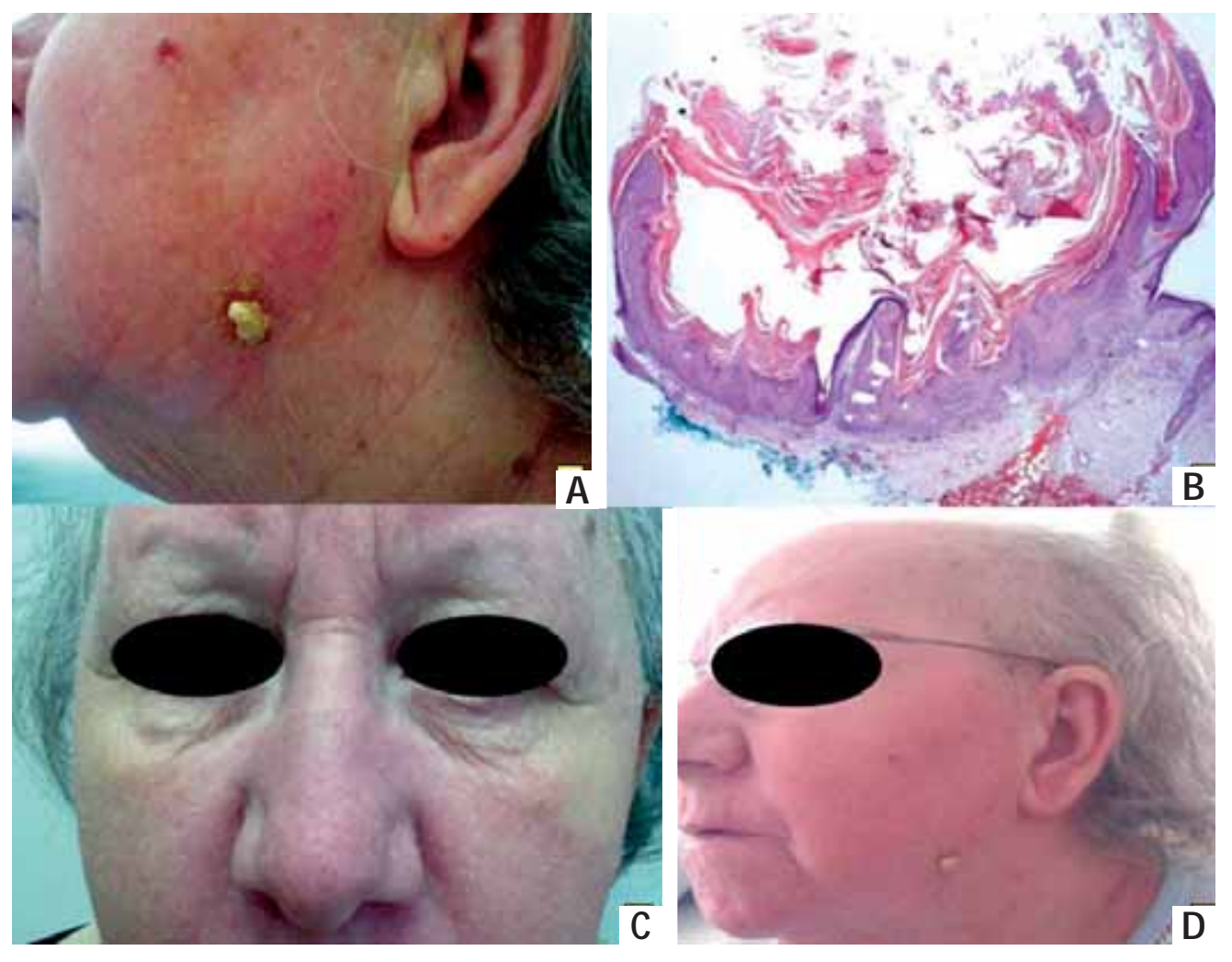

\section{Figure 1:}

Case 1: Keratoacanthoma on the left mandibula (A) confirmed by histological analysis, hematoxylin-eosin, 20x (B), photosensitivity (C) and alopecia (D)
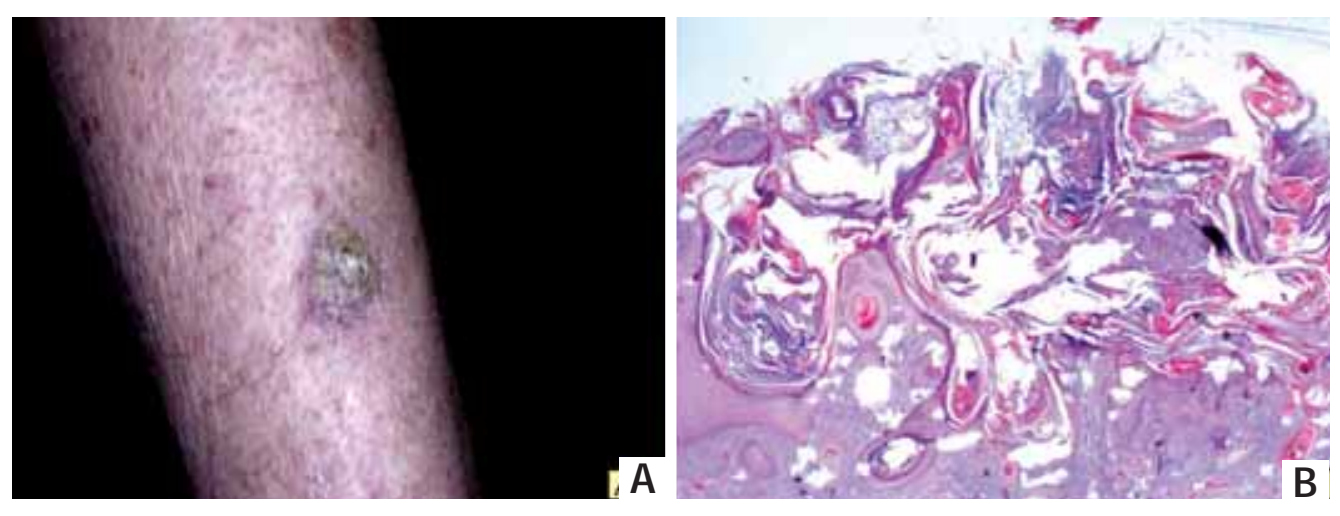

\section{Figure 2:}

Case 2: Hyperkeratotic and erithematous plaque on the left forearm developing during Vemurafenib treatment (A). Histological analysis revealing well-differentiated squamous cell carcinoma, hematoxylin-eosin, 50x (B)

\section{Case 3}

Male patient, aged 71, was diagnosed with nodular melanoma on his right arm in 2009. In October 2012, he developed metastases. In May 2013, he was started on Vemurafenib. After a month, an irregular melanocytic lesion in the abdomen was identified, which was a melanoma in situ. He endured a keratosis pilaris-like rash thinning of hair, plantar hyperkeratosis, verrucous papillomas and SCC. After 5 months of treatment, there was a dermatoscopic alteration of the nevus on the right thigh, with suspicion confocal microscopy, but the diagnosis was junctional nevus (Figure 3).

\section{Case 4}

Male patient, aged 49, was diagnosed in 2007 with superficial spreading melanoma on the back. In 2010, he was diagnosed with metastases, underwent biochemotherapy followed by ipilimumab, with no response. In February 2012, he was started on Vemurafenib. Five months later, he showed dermatoscopic alteration of the nevus on the back; the diagnosis was melanoma in situ (Figure 4). In November 2012, Vemurafenib was discontinued due to disease progression. 

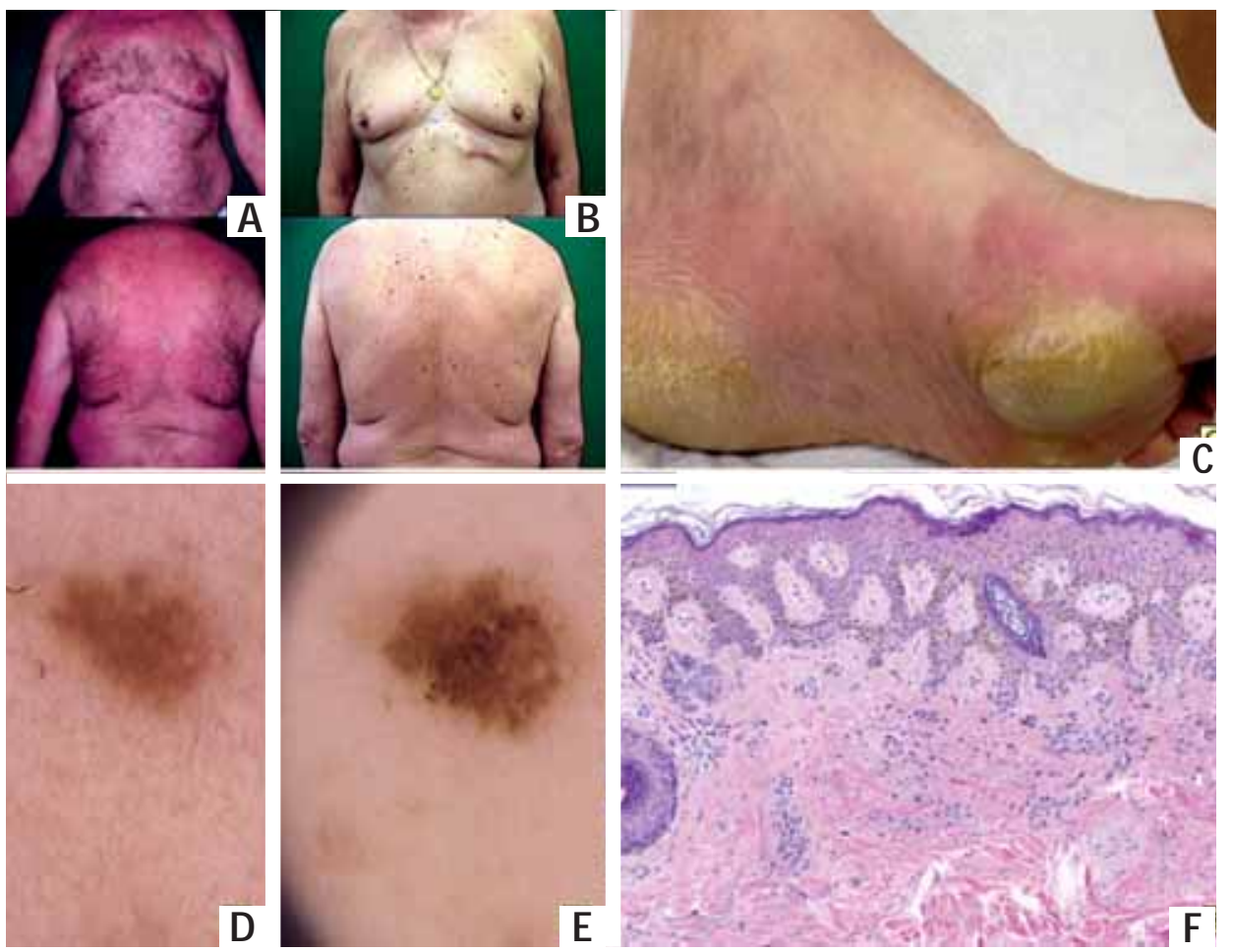

\section{Figure 3:}

Case 3: Alopecia on the trunk before $(\mathbf{A})$ and during Vemurafenib treatment (B). Hyperkeratosis of the feet (C). Dermoscopic changes in a melanocytic lesion on the right thigh, with increase in dark-brown pigmentation and globules $(\mathbf{D}, \mathbf{E})$. Histologic analysis shows a junctional nevus. Hematoxylin-eosin, 100x (F)

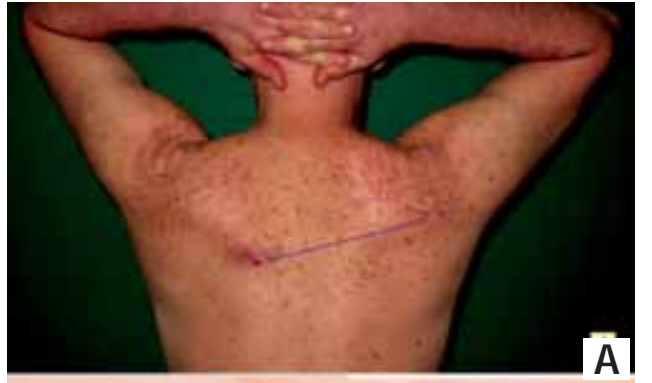

FIgURE 4:

Case 4:

Newly

developed melanocytic lesion on the upper back

(A).

Dermoscopic analysis shows irregular, dark-brown

B

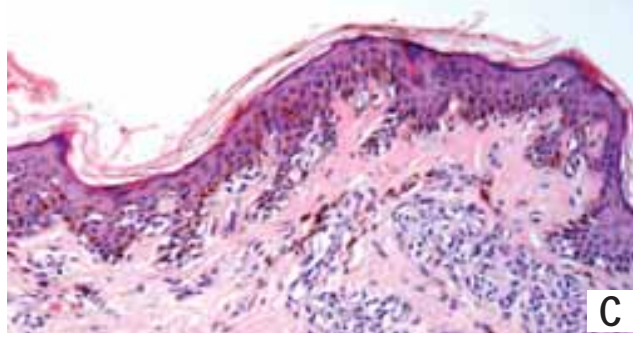
pigmentation (B), with histological features of melanoma in situ. Hematoxylin-eosin, 100x (C)

\section{Case 5}

Female patient, aged 35, was diagnosed with superficial spreading melanoma in her right leg in May 2012. She underwent wide excision, lymphadenectomy and treatment with IL-2. In May 2013, she developed brain metastasis, started radiotherapy and treatment with Vemurafenib. After 15 days, the irradiated area showed dermatitis, which improved with topical steroids. Erythematous and painful nodules appeared on the lower limbs, suggesting erythema nodosum (Figure 5). CRP, ANA, RF, and alpha-1-antitrypsin were normal and the biopsy was unsatisfactory. Oral corticosteroids were indicated for lesion control. She showed decreased and increased pigmentation of some nevi and photosensitivity (Figure 6). In December 2013, she died due to disease progression.

\section{DISAUSSION}

Vemurafenib promotes the survival of patients with metastatic melanoma ${ }^{1}$. It inhibits the MAPK pathway by binding to BRAF V600E, resulting in an antitumor effect in melanoma. ${ }^{2}$ The most frequent adverse events are cutaneous 1-3.

The proliferation of keratinocytes is common when using Vemurafenib, ranging from benign, verrucous lesions to malignant, such as SCC. This appears early, usually between the $7^{\text {th }}$ and $8^{\text {th }}$ weeks of treatment and seems to be caused by the paradoxical 

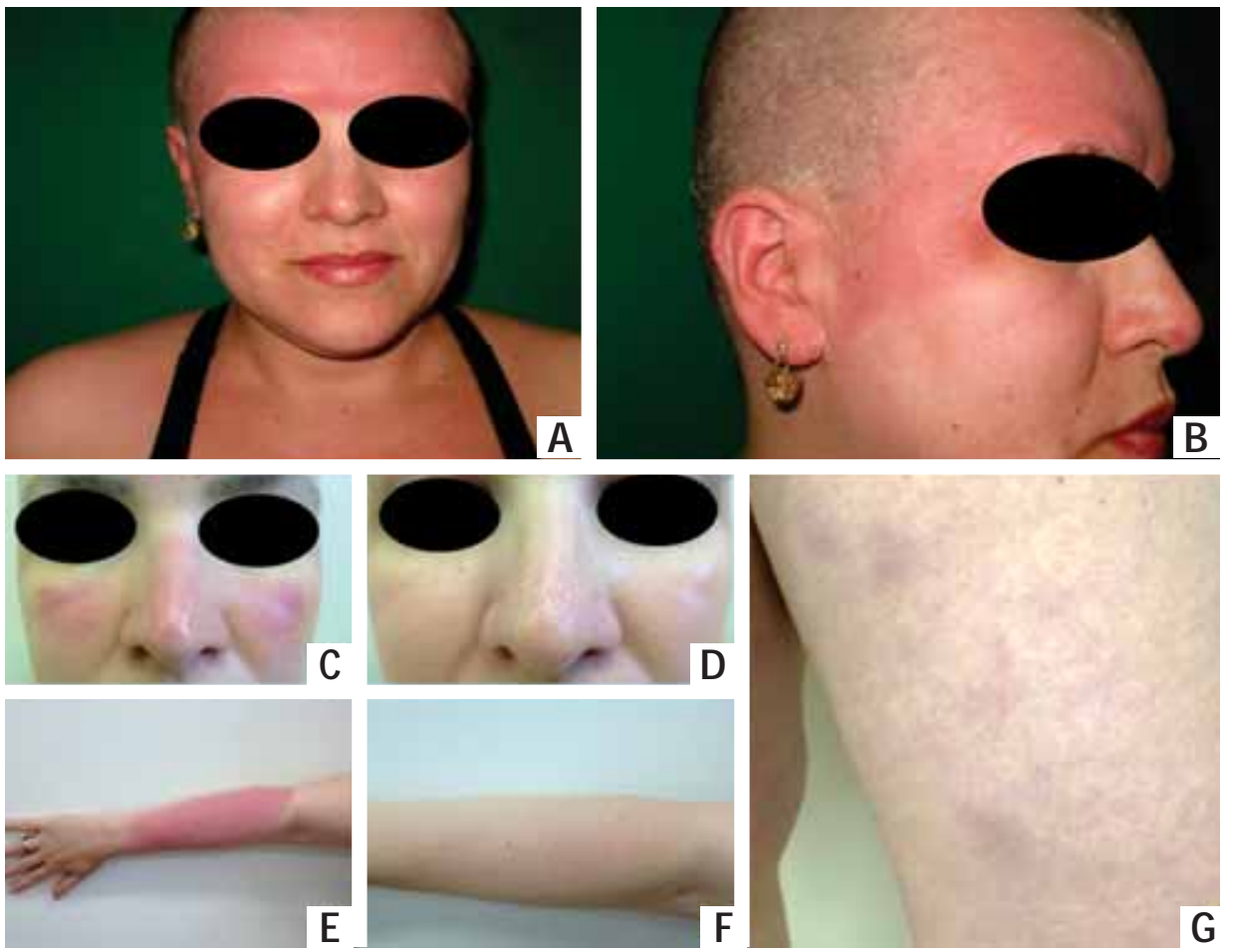

\section{Figure 5:}

Case 5: Radiation sensitivity on the scalp and face $(\mathbf{A}, \mathbf{B})$. Photosensitivity on the face $(\mathbf{C}, \mathbf{D})$ and right forearm $(\mathrm{E}, \mathrm{F})$ before and after topical corticosteroid treatment. Panniculitis on the left thigh (G)

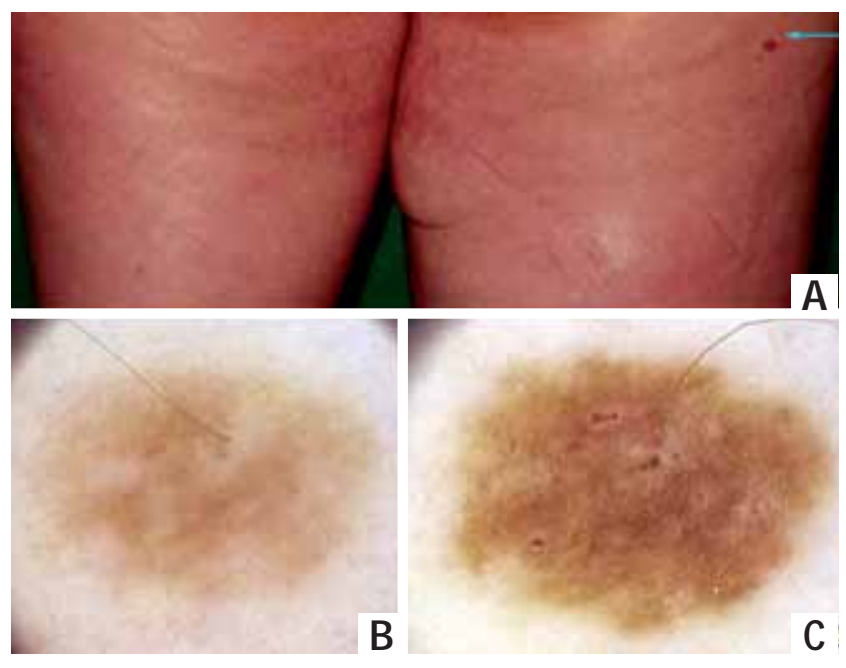

Figure 6: Case 5: Melanocytic lesion on the upper back thigh (A). Dermoscopic changes of the nevus, with increase in dark-brown pigmentation and dots (C)

activation of MAPK by Vemurafenib in cells with wild BRAF and RAS mutation through CRAF dimers. ${ }^{2,3,10} \mathrm{It}$ is believed that keratinocytes from sun-exposed areas in elderly patients with fair skin have RAS mutations and their stimulation can cause skin tumors. ${ }^{1,2,10}$

Rashes are common and can be keratosis pilaris-like or maculopapular; they usually spare the face. ${ }^{3,10}$ They may coalesce, with the appearance of toxic erythema. Normally, they are not severe enough to require discontinuation of treatment. 3,10

Photosensitivity during treatment with Vemurafenib occurs after sun exposure. UVA radiation plays an important role in pathogenesis and it permeates glass. Daily use of sunscreen (minimum SPF 30 and broad UVA protection), appropriate clothing and accessories and 100\% UVA and UVB protective films on the windows of the car, house or at work, are recommended. ${ }^{3,10}$

BRAF mutation is a common occurrence in melanocytic nevi. ${ }^{4}$ It is presumed that Vemurafenib action in these cases induces involution, whereas wild BRAF nevi would undergo paradoxical activation of MAPK pathway and atypia. ${ }^{4,5}$ A second primary melanoma caused by Vemurafenib has been reported. ${ }^{5}$ BRAF V600E metastases respond to Vemurafenib, whereas wild BRAF melanomas would be activated. ${ }^{5}$ Digital dermoscopy performed before therapy with a monthly follow-up enables identification of suspicious lesions, while confocal microscopy can be complementary. ${ }^{5}$ Skin biopsy is useful for diagnostic confirmation.

Panniculitis associated with Vemurafenib treatment has been described. 6,7 It may appear along with fever, arthritis and arthralgia. In general, biopsies reveal the presence of predominantly lobular neutrophilic panniculitis. ${ }^{7}$ It can be treated with oral corticosteroids, anti-inflammatory and discontinuation of Vemurafenib, when it is too symptomatic. Expectant 
follow-up is an option in milder cases. ${ }^{6}$

Vemurafenib can cause sensitivity in the irradiated skin and can be added to the list of drugs that trigger radiation recall dermatitis. Topical corticosteroids relieve symptoms. ${ }^{8}$ Camidge et al. suggested that skin reactions caused by drugs administered up to 7 days after radiotherapy should be considered radiosensitization reactions, rather than radiation recall. ${ }^{9}$

\section{REFERENCES}

1. Chapman PB, Hauschild A, Robert C, Haanen JB, Ascierto P, Larkin J, et al. Improved Survival with Vemurafenib in Melanoma with BRAF V600E mutation. N Engl J Med. 2011;364:2507-16.

2. Huang V, Hepper D, Anadkat M, Cornelius L. Cutaneous toxic effects associated with vemurafenib and inhibition of the BRAF pathway. Arch Dermatol. 2012;148:628-33.

3. Lacouture ME, Duvic M, Hauschild A, Prieto VG, Robert C, Schadendorf D, et al. Analysis of dermatologic events in vemurafenib-treated patients with melanoma. Oncologist. 2013;18:314-22.

4. Haenssle HA, Kraus SL, Brehmer F, Kretschmer L, Völker B, Asper H, et al. Dynamic changes in nevi of a patient with melanoma treated with vemurafenib: importance of sequential dermoscopy. Arch Dermatol. 2012;148:1183-5.

5. Dalle S, Poulalhon N, Debarbieux S, Zaharia D, Mihm MC, Lacouture ME, et al. Tracking of second primary melanomas in vemurafenib-treated patients. JAMA Dermatol. 2013;149:488-90.

6. Maldonado-Seral C, Berros-Fombella JP, Vivanco-Allende B, Coto-Segura P, Vazquez-Lopez F, Perez-Oliva N. Vemurafenib-associated neutrophilic panniculitis: An emergent adverse effect of variable severity. Dermatol Online J. 2013;19:16.

7. Zimmer L, Livingstone E, Hillen U, Dömkes S, Becker A, Schadendorf D. Panniculitis with arthralgia in patients with melanoma treated with selective BRAF inhibitors and its management. Arch Dermatol. 2012;148:357-61.

8. Boussemart L, Boivin C, Claveau J, Tao YG, Tomasic G, Routier E, et al. Vemurafenib and radiosensitization. JAMA Dermatol. 2013;149:855-7.

9. Camidge $\mathrm{R}$, Price $\mathrm{A}$. Characterizing the phenomenon of radiation recall dermatitis. Radiother Oncol. 2001;59:237-45.

10. Sinha R, Edmonds K, Newton-Bishop JA, Gore ME, Larkin J, Fearfield L. Adverse cutaneous events associated with vemurafenib in patients with metastatic melanoma: practical advice on diagnosis, prevention and management of the main treatment-related skin toxicities. Br J Dermatol. 2012;167:987-94.
Dermatologists and oncologists must be aware of skin reactions caused by Vemurafenib and schedule routine appointments during therapy. We recommend dermatologic evaluation with dermoscopy before the start of treatment, after 4 weeks and every 8 weeks, together with confocal microscopy, for better assessment of these lesions, whenever possible.

\author{
M AILING ADDRESS: \\ Giselle de Barros Silva \\ Rua Professor A ntonio Prudente, 211 \\ Liberdade \\ 01509-010 - São Paulo - SP \\ Brazil \\ E-mail: giselle_barros@yahoo.com.br
}

H ow to cite this article: Silva GB, Mendes AP, Macedo MP, Pinto CAL, Gibbons IL, Duprat Neto JP. Vemurafenib and adverse cutaneous events - report of five cases. An Bras Dermatol. 2015;90(3 Supl 1):S242-6. 